\title{
Quasi-Isotropic Cycles and Nonsingular Bounces in a Mixmaster Cosmology
}

\author{
Chandrima Ganguly ${ }^{1, *}$ and Marco Bruni ${ }^{2,3, \uparrow}$ \\ ${ }^{1}$ Department of Physics and Astronomy, Wilder Laboratories, Dartmouth College, Hanover, New Hampshire 03755, USA \\ ${ }^{2}$ Institute of Cosmology and Gravitation, University of Portsmouth, \\ Dennis Sciama Building, Burnaby Road, Portsmouth PO1 3FX, United Kingdom \\ ${ }^{3}$ INFN Sezione di Trieste, Via Valerio 2, 34127 Trieste, Italy
}

(Received 13 March 2019; revised manuscript received 11 September 2019; published 12 November 2019)

\begin{abstract}
A Bianchi type-IX mixmaster spacetime is the most general spatially homogeneous solution of Einstein's equations and it can represent the space-averaged universe. We introduce two novel mechanisms resulting in a mixmaster universe with nonsingular bounces that are quasi-isotropic. Matter with a nonlinear equation of state allows these bounces. Using a negative anisotropic stress successfully isotropizes this universe and mitigates the well-known mixmaster chaotic behavior. The Universe can be an eternal mixmaster spacetime, going through an infinite series of quasi-isotropic cycles separated by bounces.
\end{abstract}

DOI: 10.1103/PhysRevLett.123.201301

Introduction.-Cosmological parameter extraction from Planck [1-3] and other studies [4] place tight constraints on the anisotropy of the current day Universe, $\left(\sigma_{S} / H\right)_{o} \sim$ $10^{-11}$, where $\sigma_{S}$ represent the contribution of the (scalar part of) shear anisotropic expansion, and $H$ is the isotropic expansion rate. A main problem of classical cosmology is that a big bang singularity is quite ubiquitous [5-7]. Inflation through exponential expansion is able to propose a mechanism of diluting out any initial anisotropy, homogeneity, or curvature. Most importantly, the inflationary scenario is predictive, as it provides the setting to generate the observable fluctuations in the cosmic microwave background and in the matter distribution. However, there remain shortcomings within this paradigm. For example, many models cannot be extrapolated backward, unable to escape a big bang. The energy scale of inflation can also be high enough for a complete theory of quantum gravity to be required [8]. All cosmologically interesting fluctuation modes may have originated from a trans-Planckian zone of ignorance-the physics of which would significantly impact the predicted spectrum of cosmological perturbations [9].

The scenario proposed in this Letter does away with this problem by producing an alternate "beginning" story to the observable Universe- a successful bounce that solves the issue of the big bang singularity and the trans-Planckian problem [10], followed by a quasi-isotropic expansion.

We assume general relativity (with units $c=8 \pi G=1$ ) and a matter source with a nonlinear equation of state (EOS) $[11,12]$

$$
P=P_{o}+\alpha \rho+\frac{\epsilon}{\rho_{c}} \rho^{2},
$$

relating the pressure $P$ and the energy density $\rho$. For the most general case of an anisotropic fluid, conservation of energy is guaranteed by

$$
\dot{\rho}=-3 H(\rho+P)-\pi_{\mu \nu} \sigma^{\mu \nu},
$$

where $\dot{\rho} \equiv u^{\alpha} \nabla_{\alpha} \rho$ is the proper time derivative along the fluid flow lines with 4-velocity $u^{\alpha}, 3 H=\nabla_{\alpha} u^{\alpha}$ is the expansion of the fluid, $\sigma_{\mu \nu}$ is its shear, and $\pi_{\mu \nu}$ is the anisotropic stress [7]. Here $\rho_{c}$ is the characteristic energy scale at which the effect of the nonlinearities in the EOS becomes relevant. $P_{o}$ can play the role of an effective cosmological constant [12]; in this Letter, we assume $P_{o}=0$ and $\epsilon=-1$. Then, for the perfect fluid case, this gives an effective cosmological constant

$$
\rho_{\Lambda}=(\alpha+1) \rho_{c}
$$

i.e., a stationary point of (2), so that it must be $\rho<\rho_{\Lambda}$, or otherwise one gets a "phantom behavior," i.e., $\dot{\rho}>0$ during expansion $(H>0)$ [12]. In other words, in our scenario we have a high-energy cosmological constant for $\pi_{\mu \nu}=0$. Choosing $\alpha=1 / 3$ to correspond to the radiation case at low energies, when the quadratic term is negligible, we have therefore a maximum value $\rho_{\Lambda}=4 \rho_{c} / 3$ for $\rho$. A closed (positively curved space) Friedmann-LemaîtreRobertson-Walker (FLRW) universe with this EOS can undergo cycles with nonsingular bounces, as shown in [12]; here we want to use this EOS in the context of a Bianchi type-IX cosmology, generalizing [11,12]. In this scenario, as long as $\rho_{\Lambda}$ is below the Planck energy scale, the universe undergoes a bounce at energies where one can use classical general relativity. Other components such as standard matter can be introduced, but here we restrict the analysis to the bare bones of the scenario, to make it simpler.

The next question addressed in this Letter is that of growing anisotropies in the contracting phase of a bouncing cosmology. We introduce a novel isotropization mechanism using a negative anisotropic stress. This can represent shear 
viscosity and has been studied in expanding universes in [13]. A negative $\pi_{\mu \nu}$ can represent viscous effects and successfully mitigate the growth of expansion anisotropy, as well as the chaotic behavior that is ubiquitous in spatially homogeneous spacetimes with anisotropic 3-curvature. This is an alternative to the "ultrastiff" linear EOS $p \gg \rho$ that has been used in the literature so far $[14,15]$.

The Bianchi type-IX universe.-To understand the behavior of the most general kind of anisotropies-both in expansion as well as in the 3-curvature-we turn to the Bianchi type-IX models. These are the most general anisotropic, spatially homogeneous spacetimes [16]. When one considers solutions of Einstein's equations of this type, under standard assumption on the matter content [5], typically these models approach a singularity where matter becomes negligible, with the anisotropic 3-curvature driving the scale factors to undergo infinite chaotic oscillations over a finite time interval [16-19]. This chaotic behavior is actually an attractor, called the mixmaster attractor [20]. This behavior has been studied numerically in [21-23] among others. An important reason why this class of cosmological models is interesting is because they contain the closed FLRW model as their isotropic subcase. Universe models having positive spatial curvature have always been of particular interest, as cycling solutions have been found in the isotropic case sourced by a linear EOS fluid $[12,24]$ and by our present quadratic EOS [12]. In the Bianchi type-IX models, the sign of the 3-curvature is crucial: only when they are close enough to isotropy the 3-curvature is positive and reexpansion can occur. Sufficient isotropization must therefore take place before the universe can reexpand after collapse. Attempts at isotropizing the Bianchi type-IX cosmology and mitigating the chaotic behavior have mostly been focused on introducing stiff $(P=\rho)[25]$ or superstiff $(P \gg \rho)$ matter $[14,15,26,27]$. Adding a superstiff fluid does not always seem to work, as the existence of superstiff anisotropic stress causes a faster growth of the shear anisotropies [28]. We study the effects of the nonlinear EOS (1) and the negative anisotropic stress by numerically integrating the Einstein field equations and the conservation equation for the energy momentum tensor $T_{\nu}^{\mu}$ for these cosmological models.

In general, the metric for a homogeneous spacetime is given by

$$
d s^{2}=-d t^{2}+\gamma_{a b} \omega^{a} \omega^{b} .
$$

Here $\omega^{a}$ are the 1-forms for the triad basis in which the spacetime is defined. They are general functions of the spatial coordinates. The $\gamma_{a b}$ are the metric components in this triad and are functions of time only, as the spacetime is homogeneous. For our purposes, the metric in the triad frame is explicitly given by $\gamma_{a b}=$ diagonal matrix $\left[a(t)^{2}, b(t)^{2}, c(t)^{2}\right]$, where $a(t), b(t)$, and $c(t)$ will be taken to be the dimensionless scale factors of the universe in the three spatial directions. The isotropic FLRW subcase is given by $a(t)=b(t)=c(t)$.

We can write the equations of motion for this system in the triad basis in terms of $\gamma_{a b}$ and its time derivative, the extrinsic curvature $\kappa_{a b}=(\partial / \partial t) \gamma_{a b}$ [16]. Thus, mathematically, the problem is reduced to the study of a nonlinear dynamical system of ordinary differential equations.

The Bianchi type-IX universe with matter has been found to undergo rotation of the triad frame axes themselves. The triad frame itself is part of the dynamics and the rotation can be parametrized in terms of the Euler angles $\theta$, $\phi$, and $\psi$. For our present purposes, however, we are not interested in matter that exhibits noncomoving velocities or vorticities (see [29] and references therein). Thus the stressenergy tensor $T_{\nu}^{\mu}$ is diagonal. Einstein equations then imply that the nondiagonal components of the Ricci tensor are zero. It has been shown [5] that this also implies that the Euler angles $\theta, \phi$, and $\psi$ are constant and frame rotation can be disregarded in this case.

We also introduce the following variables:

$$
3 x=\frac{\dot{a}}{a}-\frac{\dot{b}}{b}, \quad 3 y=\frac{\dot{a}}{a}-\frac{\dot{c}}{c}, \quad 3 H=\frac{\dot{a}}{a}+\frac{\dot{b}}{b}+\frac{\dot{c}}{c} .
$$

These variable definitions are useful: $3 H$ gives the overall expansion of the volume of the universe, and $x$ and $y$ are directly related to the shear $\sigma$ (made of the logarithmic derivatives of $a, b$, and $c$ ). They allow us to track the growth of anisotropy. We choose a fluid 4-velocity $u^{\mu}=$ $(1,0,0,0)$ and consider a diagonal stress-energy tensor given by $T_{\nu}^{\mu}=$ diagonal matrix $[-\rho, P, P, P]$, where $P(\rho)$ is given by (1). Therefore, using Eq. (1) in (2), together with Eqs. (5) and three equations for $x, y$, and $H$, we have a dynamical system consisting of seven coupled first-order ordinary differential equations for seven variables.

Characteristic scale of the problem.-The nonlinear EOS (1) is characterized by the energy scale $\rho_{c}$. We can then introduce new dimensionless variables

$$
\tilde{x}=\frac{x}{\sqrt{\rho_{c}}}, \quad \tilde{y}=\frac{y}{\sqrt{\rho_{c}}}, \quad \tilde{H}=\frac{H}{\sqrt{\rho_{c}}}, \quad \tilde{\rho}=\frac{\rho}{\rho_{c}},
$$

which amounts to introduce a dimensionless time $\eta=\sqrt{\rho_{c}} t$ [12]. Then, rewriting time derivatives in the equations of motion in terms of $\eta$, the dynamics can made completely dimensionless and independent from $\rho_{c}$. Equivalently, for the purposes of our computations, we can use $\rho_{c}$ as our unit, setting $\rho_{c}=1$ in our equations. However, $\rho_{c}$ is related to the initial conditions and needs to be reintroduced in order to get physical results. We will comment on the effect of this in a later section.

The numerical integration of Einstein's equations in this Letter has been carried out using Wolfram's Mathematica and using the LSODE solver-this alternates between 


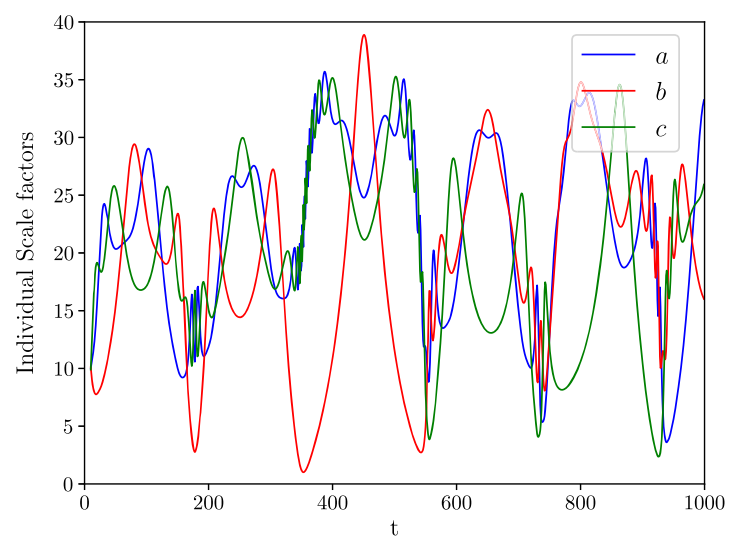

FIG. 1. Blue, orange, and green lines denote the scale factors $a$, $b$, and $c$, normalized to the curvature scale. The model is sourced by the nonlinear equation of state $\frac{1}{3} \rho-\rho^{2}$, with no anisotropic stress. Time is expressed in units of $\rho_{c}$.

Adams method in the nonstiff case (when the system has sufficiently isotropized) and the backward differential formula method for the stiff case when the system is highly anisotropic and oscillations are rapid.

Results from introducing only the nonlinear fluid.-The results of the numerical integration in Fig. 1 reveal that the individual scale factors undergo several oscillations as expected and the volume scale factor undergoes bounces of similar height. This behavior is reminiscent of the analysis done by [23] for linear equations of state, while the negative quadratic term in Eq. (1) is similar to the effective term in loop quantum cosmology [30]. The shear and the 3-curvature shoot up at the minima but remain small when the universe is at its maximum size. The energy density in the anisotropies $\sigma^{2}$ is diluted by expansion and is the smallest at the expansion maxima and shows peaks near the minima. However, we see from Fig. 2 that the evolution of the shear energy density has chaotic peaks. This implies that, although the nonlinear EOS (1) is successful in creating a nonsingular bounce, it is not as successful in isotropizing the universe, in contrast with the flat Bianchi type-I case studied in [31]. This bouncing universe does not seem capable of satisfying the stringent observational constraints on isotropy.

Introduction of negative anisotropic stress as a mechanism of isotropization.-The anisotropy energy density given by $\sigma^{2} \sim \sigma_{a b} \sigma^{a b}$ grows as (volume) $)^{-2}$. The solution proposed by ekpyrosis [32-34] is to introduce a scalar field rolling down a steep negative exponential potential. The idea is that this field-also known as the ekpyrotic field-will have an effective EOS that is superstiff, i.e., $P \gg \rho$. This effective superstiff fluid will evolve much faster than (volume $)^{-2}$ in a universe with contracting volume, and so will be able to dominate over the anisotropy and inhomogeneity in the contracting phase of a bouncing universe. When anisotropic stresses are included, which are themselves superstiff, they act as a source for growing

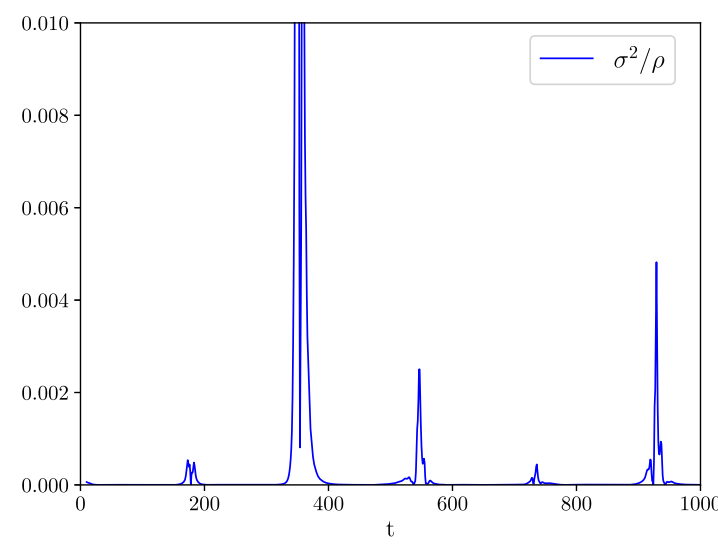

FIG. 2. Plot of the normalized dimensionless shear $\sigma^{2} / \rho$. The model is sourced by the nonlinear equation of state $\frac{1}{3} \rho-\rho^{2}$, with no anisotropic stress.

shear, and hence any ekpyrotic field will always have to compete with these growing anisotropies. This has been studied in detail in [28]. Furthermore, a universe that is highly anisotropic and has nonzero spatial curvature-like the Bianchi type-IX universe-will not reexpand through a bounce after contraction, as the anisotropies will dominate over the fields that drive the bounce [35]. In the specific case of the Bianchi type-IX universe, this will signal the onset of chaotic behavior. By the same reasoning, a negative anisotropic stress should cause the shear to decrease. This has been seen in the context of shear viscosity in expanding anisotropic universes in [36].

We investigate whether similar forms of negative anisotropic stress can be used as a novel isotropization mechanism in the presence of both expansion as well as curvature anisotropies. We choose the form of the anisotropic stress to be

$$
\pi_{a b}=\kappa \rho^{1 / 2} \sigma_{a b},
$$

where $\kappa$ is a dimensionless constant that we choose to be negative $\kappa<0$. This form of the anisotropic stress is also useful, as the effect of these stresses only become significant at sufficiently high energies near the bounce. The negative proportionality constant should lead to the reduction of the shear without having to take recourse by introducing a superstiff fluid. The evolution equation for the shear tensor in a flat Bianchi type-I universe in the presence of anisotropic stress illustrates this fact,

$$
\dot{\sigma}_{a b}+3 H \sigma_{a b}=\pi_{a b},
$$

where $\pi_{a b}$ is the anisotropic stress.

The stress-energy tensor now becomes $T_{\nu}^{\mu}=$ diagonal $\operatorname{matrix}\left[-\rho, p+\pi_{11}, p+\pi_{22}, p+\pi_{33}\right]$.

We now solve the Einstein field equations in the Bianchi type-IX universe with the inclusion of this anisotropic stress. We find that the bouncing behavior is still sustained 


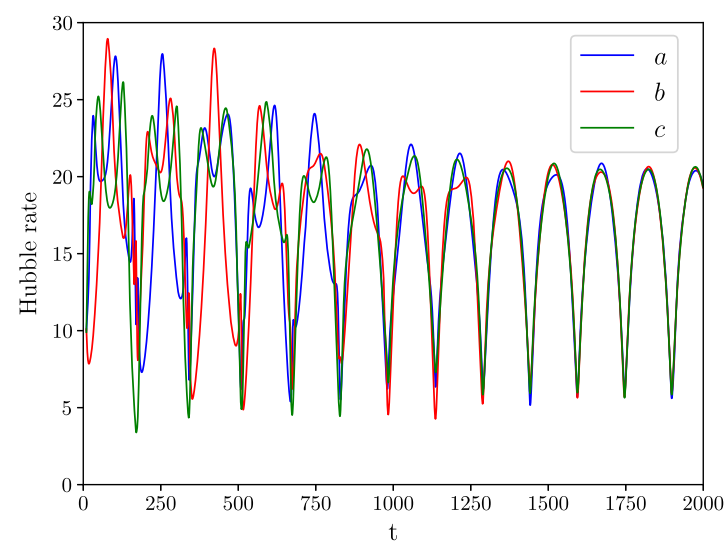

FIG. 3. Blue, orange, and green lines denote the scale factors $a$, $b$, and $c$, normalized to the curvature scale. Anisotropic stress $\pi_{a b}=\kappa \sqrt{\rho} \sigma_{a b}$ has been added to the fluid, in addition to the nonlinear equation of state for the isotropic pressure that was sourcing the model before. $\kappa=-4$ in this computation. Time is expressed in units of $\rho_{c}$.

with the bounces becoming more isotropic, see Fig. 3 . Furthermore we find, as shown in Fig. 4, that the peaks in the shear at the expansion minima of the model now decrease in height over time. This seems to point to isotropy being achieved. It now remains to be seen if the chaotic behavior that is an attractor in the Bianchi type-IX universe is also suppressed.

Effect on mixmaster chaos. - The onset of chaotic behavior in the mixmaster system has been studied analytically, numerically, and with invariant methods, e.g., see $[14,23,37,38]$ and references therein. By chaotic behavior, we mean that, for a small change in initial conditions, the solution trajectories diverge exponentially from one another during the course of the evolution, eventually filling the phase space. The principal Lyapunov exponent measures the rate at which the solution trajectories begin to

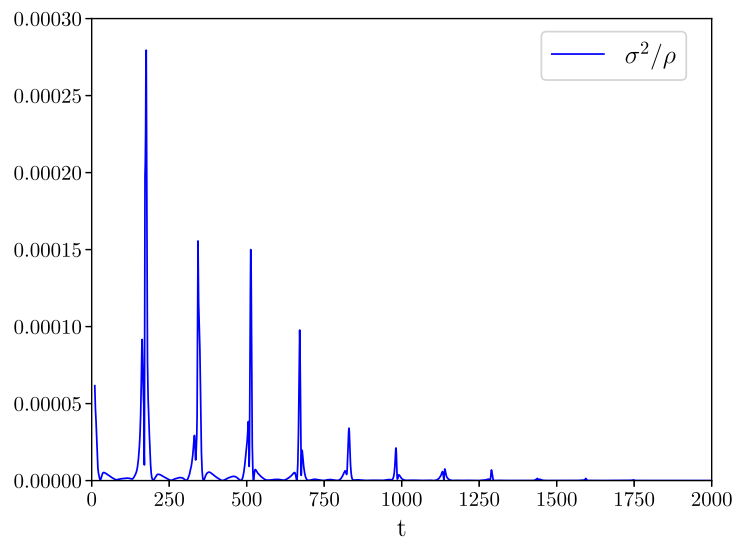

FIG. 4. Normalized dimensionless shear $\sigma^{2} / \rho$ for a model sourced by quadratic equation of state $P=\frac{1}{3} \rho-\rho^{2}$ with the inclusion of anisotropic stress $\pi_{a b}=\kappa \rho^{1 / 2} \sigma_{a b} . \kappa=-4$ in this computation. diverge. A positive principal Lyapunov index would signify the onset of chaos, while, vice versa, a negative principal Lyapunov index signals the suppression of chaos. We choose initial conditions containing some initial expansion as well as 3-curvature anisotropy, but with this being small enough to resemble the isotropic Friedmann universe very closely. We then evaluate the time evolution of the principal Lyapunov index numerically to find that the mixmaster chaos is completely mitigated, as the principal Lyapunov index is negative and of the order of -2 . It is interesting to note that this happens even without the inclusion of a stiff fluid as observed in [25] or a superstiff field as shown in [14]. This is caused by the reduction in shear by the inclusion of the negative anisotropic stress as well as by the nonlinear term in Eq. (1): at energies close enough to $\rho_{c}$ this term dominates and acts like an effective superstiff fluid.

Energy scale $\rho_{c}$ and size of the universe at the bounce.In our scenario for the evolution of the universe, the nonlinearity of the EOS (1) depends critically from $\rho_{c}$. The effect of increasing its value is simply to increase the time period of the oscillations, and hence the amplification factor of the volume. Since our Bianchi type-IX model evolves toward the corresponding FLRW closed model [12], we can use this to estimate the size of the universe at the bounce. Working now with a dimensionless scale factor $a(t)$ normalized to $a_{o}=1$ today and restoring units, from [12] its minimum value $a_{m}$ at the bounce is at least

$$
a_{m} \geq\left(\frac{3\left|\Omega_{K}\right| H_{o}^{2} c^{2}}{8 \pi G(1+\alpha) \rho_{c}}\right)^{1 / 2}
$$

for $\rho$ bounded by $\rho_{\Lambda}$ [defined in Eq. (3)], where $K=$ $H_{o}^{2}\left|\Omega_{K}\right|$ is the curvature of the Universe, and $H_{o}$ and $\Omega_{K}$ are the today's measured Hubble parameter and curvature density parameters, respectively. The ratio of the size of the universe today and its minimum is simply $a_{o} / a_{m}=a_{m}^{-1}$ and, if in turn we assume that $\rho_{\Lambda}$ is bounded by the Planck energy density, this ratio is of the order of

$$
\frac{a_{o}}{a_{m}} \lesssim\left(\frac{8 \pi c^{5}}{3\left|\Omega_{K}\right| \hbar G H_{o}^{2}}\right)^{1 / 2} .
$$

This estimate would change with the inclusion of other matter components.

Conclusion.-In this Letter, we have studied Bianchi type-IX universe models sourced by a nonlinear equation of state fluid. We find that with a negative quadratic term in the EOS (1) we can produce a series of nonsingular bounces. These bounces and the subsequent cycles are reminiscent of the mixmaster oscillations, which are known to be chaotic in cases when the universe is sourced by fields obeying the strong energy condition (SEC), see [39] for an example. In our scenario, the field obeys the SEC at low energies, but at energies close enough to $\rho_{\Lambda}$ the nonlinear 
term that drives the bounce becomes important and the SEC can be violated. Crucially, the null energy condition is not violated and $\dot{\rho}<0$ during expansion, avoiding a phantom behavior [40]. If the energy scale $\rho_{\Lambda}$ is sufficiently smaller than the Planck scale, the evolution is purely classical and no quantum gravity corrections are needed.

We go a step further to propose a novel mechanism of isotropizing the anisotropic closed Bianchi type-IX universe models by introducing a negative anisotropic stress. The effect is isotropization as well as the mitigation or even suppression of the well-known mixmaster chaos. This mechanism does not take recourse to the use of a superstiff fluid reminiscent of the ekpyrosis scenario [33,41].

The net result of introducing the nonlinear EOS (1) together with a negative anisotropic stress (2) is that we obtain Bianchi type-IX cycling universe models that go through bounces followed by expansion and recontraction, while remaining close to a FLRW model with positive spatial curvature during expansion epochs. Thus, these models, the most general homogeneous solutions of Einstein equations, should satisfy observational constrains [1-3], specifically those on anisotropy [4], once all relevant matter components are included.

For simplicity, here we have used a single fluid representing radiation at low energies, to demonstrate the mechanism of the bounce and isotropization. Our results generalize easily to the inclusion of more matter components, in particular, a standard pressureless matter (giving a matter-dominate era) and dark energy, which are both subdominant to radiation and negligible once the evolution is close to a bounce. In future work, we will consider more general nonlinear EOS such as the Shan-Chen EOS [42] used in [43] and we will include a standard matter component, also investigating the effects of various dark energy scenarios on the type of cosmological models considered here. It is also important to study the stability of our solution to inhomogeneities - such as those studied in [44-46], among others.

C. G. would like to acknowledge Marcelo Gleiser and John D. Barrow for many useful discussions. C. G. was supported by the Lindemann Fellowship and was hosted at Dartmouth College. M. B. is supported by UK STFC Grant No. ST/N000668/1.

*ganguly.chandrima@gmail.com †marco.bruni@port.ac.uk

[1] Planck Collaboration, Planck 2015 results - xiii. Cosmological parameters, Astron. Astrophys. 594, A13 (2016).

[2] Planck Collaboration, Planck 2015 results - xvi. Isotropy and statistics of the cmb, Astron. Astrophys. 594, A16 (2016).

[3] Planck Collaboration, Planck 2015 results - xviii. background geometry and topology of the universe, Astron. Astrophys. 594, A18 (2016).
[4] D. Saadeh, S. M. Feeney, A. Pontzen, H. V. Peiris, and J. D. McEwen, How Isotropic is the Universe?, Phys. Rev. Lett. 117, 131302 (2016).

[5] V. Belinski and M. Henneaux, The cosmological singularity, in Cambridge Monographs on Mathematical Physics (Cambridge University Press, Cambridge, England, 2017).

[6] S. Hawking and W. Israel, General Relativity: An Einstein Centenary Survey (2010).

[7] S. W. Hawking and G. F. R. Ellis, The large scale structure of space-time, in Cambridge Monographs on Mathematical Physics (Cambridge University Press, Cambridge, England, 2011).

[8] A. R. Liddle, The inflationary energy scale, Phys. Rev. D 49, 739 (1994).

[9] J. Martin and R.H. Brandenberger, The trans-Planckian problem of inflationary cosmology, Phys. Rev. D 63, 123501 (2001).

[10] R. Brandenberger and P. Peter, Bouncing cosmologies: Progress and problems, Found. Phys. 47, 797 (2017).

[11] K. N. Ananda and M. Bruni, Cosmo-dynamics and dark energy with a quadratic EoS: Anisotropic models, largescale perturbations and cosmological singularities, Phys. Rev. D 74, 023524 (2006).

[12] K. N. Ananda and M. Bruni, Cosmo-dynamics and dark energy with non-linear equation of state: A quadratic model, Phys. Rev. D 74, 023523 (2006).

[13] C. W. Misner, The isotropy of the universe, Astrophys. J. 151, 431 (1968).

[14] A. B. Burd, N. Buric, and G. F. R. Ellis, A numerical analysis of chaotic behaviour in Bianchi IX models, Gen. Relativ. Gravit. 22, 349 (1990).

[15] J. E. Lidsey, Cosmic no hair for collapsing universes, Classical Quantum Gravity 23, 3517 (2006).

[16] L. D. Landau, Textbook on Theoretical Physics. Vol. 2: Classical Field Theory (Pergamon, Oxford, 1975).

[17] V. A. Belinsky, I. M. Khalatnikov, and E. M. Lifshitz, Oscillatory approach to a singular point in the relativistic cosmology, Adv. Phys. 19, 525 (1970).

[18] M. Bruni and C. F. Sopuerta, Covariant fluid dynamics: A long wavelength approximation, Classical Quantum Gravity 20, 5275 (2003).

[19] N. Deruelle and D. Langlois, Long wavelength iteration of Einstein's equations near a space-time singularity, Phys. Rev. D 52, 2007 (1995).

[20] C. W. Misner, Mixmaster Universe, Phys. Rev. Lett. 22, 1071 (1969).

[21] B. K. Berger, D. Garfinkle, and E. Strasser, New algorithm for mixmaster dynamics, Classical Quantum Gravity 14, L29 (1997).

[22] K. Ferraz and G. Francisco, Mixmaster numerical behavior and generalizations, Phys. Rev. D 45, 1158 (1992).

[23] A. Zardecki, Modeling in chaotic relativity, Phys. Rev. D 28, 1235 (1983).

[24] J. D. Barrow and C. G. Tsagas, On the stability of static ghost cosmologies, Classical Quantum Gravity 26, 195003 (2009).

[25] V. A. Belinski and I. M. Khalatnikov, Effect of scalar and vector fields on the nature of the cosmological singularity, Sov. Phys. JETP 36, 591 (1973). 
[26] D. Garfinkle, W. C. Lim, F. Pretorius, and P. J. Steinhardt, Evolution to a smooth universe in an ekpyrotic contracting phase with $w>1$, Phys. Rev. D 78, 083537 (2008).

[27] K. J. Erickson, H. D. Wesley, J. P. Steinhardt, and N. Turok, Kasner and mixmaster behavior in universes with equation of state $w>1$, Phys. Rev. D 69, 063514 (2004).

[28] J. D. Barrow and C. Ganguly, Evolution of initially contracting Bianchi class A models in the presence of an ultrastiff anisotropic pressure fluid, Classical Quantum Gravity 33, 125004 (2016).

[29] C. Ganguly and J. D. Barrow, Evolution of cyclic mixmaster universes with noncomoving radiation, Phys. Rev. D 96, 123534 (2017).

[30] I. Agullo and P. Singh, Loop quantum cosmology, in Loop Quantum Gravity: The First 30 Years, edited by A. Ashtekar and J. Pullin (2017), pp. 183-240.

[31] V. Bozza and M. Bruni, A solution to the anisotropy problem in bouncing cosmologies, J. High Energy Phys. 10 (2009) 014.

[32] R. Kallosh, J. U. Kang, A. Linde, and V. Mukhanov, The new ekpyrotic ghost, J. Cosmol. Astropart. Phys. 04 (2008) 018.

[33] J. Khoury, B. A. Ovrut, N. Seiberg, P. J. Steinhardt, and N. Turok, From big crunch to big bang, Phys. Rev. D 65, 086007 (2002).

[34] P. Steinhardt and N. Turok, A cyclic model of the universe, Science 296, 1436 (2002).

[35] D. Battefeld and P. Peter, A critical review of classical bouncing cosmologies, Phys. Rep. 571, 1 (2015).
[36] L. S. Parnovskii, Influence of viscosity on the evolution of a Bianchi type II universe, Zh. Eksp. Teor. Fiz. (1977).

[37] D. F. Chernoff and J. D. Barrow, Chaos in the Mixmaster Universe, Phys. Rev. Lett. 50, 134 (1983).

[38] C. Cherubini, D. Bini, M. Bruni, and Z. Perjes, The speciality index as invariant indicator in the BKL mixmaster dynamics, Classical Quantum Gravity 22, 1763 (2005).

[39] G. F. R. Ellis, E. Platts, D. Sloan, and A. Weltman, Current observations with a decaying cosmological constant allow for chaotic cyclic cosmology, J. Cosmol. Astropart. Phys. 04 (2016) 026.

[40] K. N. Ananda and M. Bruni, Cosmological dynamics and dark energy with a nonlinear equation of state: A quadratic model, Phys. Rev. D 74, 023523 (2006).

[41] P. J. Steinhardt and N. Turok, Cosmic evolution in a cyclic universe, Phys. Rev. D 65, 126003 (2002).

[42] X. Shan and H. Chen, Lattice Boltzmann model for simulating flows with multiple phases and components, Phys. Rev. E 47, 1815 (1993).

[43] D. Bini, A. Geralico, D. Gregoris, and S. Succi, Dark energy from cosmological fluids obeying a Shan-Chen nonideal equation of state, Phys. Rev. D 88, 063007 (2013).

[44] J. D. Barrow and A. Paliathanasis, Cyclic Szekeres universes, Eur. Phys. J. C 79, 379 (2019).

[45] Ch. Charach, Electromagnetic Gowdy universes, Phys. Rev. D 19, 3516 (1979).

[46] G. F. R. Ellis and M. A. H. MacCallum, A class of homogeneous cosmological models, Commun. Math. Phys. 12, 108 (1969). 